\title{
Comparative Analysis of Robotic-Assisted Partial Nephrectomy Versus Open Partial Nephrectomy During the Initial Robotic Learning Curve: Does the End Justify the Means?
}

\author{
Ragheed Saoud ${ }^{\mathrm{a}}$, Albert El Hajj ${ }^{\mathrm{a}}$, Mohammed Shahait ${ }^{\mathrm{a}}$, Muhammad Bulbul ${ }^{\mathrm{a}}$, Rami Nasr ${ }^{\mathrm{a}}$, \\ Wassim Wazzan ${ }^{\text {a }}$, Mark Khaulia ${ }^{\mathrm{a}}$, Rana Abu Dargham ${ }^{\mathrm{a}}$, Aline Yacoubian ${ }^{\mathrm{a}}$, Raja Khauli ${ }^{\mathrm{a}}$ b
}

\begin{abstract}
Background: Several studies have alluded to a detrimental impact of the surgeon's "learning curve" on outcomes of minimally invasive surgery. In this study, we evaluated the outcomes of robotic-assisted partial nephrectomy (RAPN) versus open partial nephrectomy (OPN) for kidney tumors, during the introduction of Robotic Urologic Oncology at our institution.
\end{abstract}

Methods: A retrospective review of all consecutive partial nephrectomies (PN), RAPN and OPN, performed at the American University of Beirut Medical Center since the inception of the robotic program in July 2013 until July 2015. Thirty-four consecutive patients underwent PNs, 19 OPN and 15 RAPN. Preoperative variables (patient characteristics, tumor size, and RENAL score) and perioperative renal functional/patient outcomes (\% change in glomerular filtration rate (GFR), ischemia time, blood loss, need for blood transfusions, total operating time, and length of hospital stay) were compared using SPSS.

Results: Preoperative variables, including the size and RENAL score of the tumor were analyzed. The difference in the median size of the tumor between OPN and RAPN was not statistically significant $(4.5 \pm 2.7 \mathrm{~cm}$ vs. $3.6 \pm 1.7 \mathrm{~cm}$, respectively, $\mathrm{P}=0.25)$. RENAL score was significantly higher for OPN compared to RAPN (7.3 \pm 2.3 vs. $4.9 \pm 1.5$, respectively, $\mathrm{P}<0.05)$. Mean operative time was significantly shorter for OPN vs. RAPN (178 \pm 52 min vs. $296 \pm 86$ min, respectively, $\mathrm{P}<0.05$ ). Cold ischemia time was $24 \pm 3 \mathrm{~min}$ in OPN, and warm ischemia time was $17.5 \pm 2$ min for RAPN; 10 out of the total 15 robotic cases were performed with a warm ischemia time of $<20 \mathrm{~min}$. Intraoperative blood loss was comparable for both approaches ( $225 \pm 132 \mathrm{~mL}$ in OPN vs. $243 \pm 192 \mathrm{~mL}$ in RAPN), and there was no need for blood transfusions in either group. Hospital

Manuscript accepted for publication December 28, 2016

aAmerican University of Beirut Medical Center, Beirut, Lebanon

${ }^{b}$ Corresponding Author: Raja Khauli, American University of Beirut Medical Center, Beirut, Lebanon. Email: rkhauli@aub.edu.lb

doi: https://doi.org/10.14740/wjnu286w stay was significantly longer for OPN vs. RAPN ( $6 \pm 1.6$ days vs. $4 \pm$ 0.9 days, respectively, $\mathrm{P}=0.01)$. The change in GFR was comparable among both procedures (OPN $=-9 \%$ vs. $\mathrm{RAPN}=-7 \%)$; pathological margin status was also comparable among both procedures, with $1 / 19(5 \%)$ positive focal margins in OPN vs. $0 / 14$ in RAPN. None of the robotic procedures required conversion to the laparoscopic or open approach.

Conclusions: RAPN is currently an established approach for the treatment of kidney tumors with the advantages of decreased crude ischemia time and a shorter hospital stay, with comparable intraoperative blood loss and risk of GFR reduction. Our data show that tumor characteristics were not equivalent, with higher RENAL scores noted in patients allocated to OPN vs. RAPN, thus limiting a fair comparison of outcomes. However, the data confirm that with proper selection of patients for RAPN, outcomes were equivalent to OPN and were not jeopardized during the initial robotic learning curve. Larger prospective studies are needed to validate our results.

Keywords: Robotic; Partial; Nephrectomy; Open; Ischemia time; Learning; Curve; RENAL score

\section{Introduction}

Over the past 15 years, partial nephrectomy (PN) has become the standard of care for surgical removal of small renal masses in surgically fit patients. PN has proven to be advantageous when compared to radical nephrectomy with regard to the lower risk of azotemia [1,2], long-term renal insufficiency, and decreased long-term risk of cardiac morbidity [2]. Robotic-assisted partial nephrectomy (RAPN) has become the gold standard treatment [3] for such masses as a result of recent advances in laparoscopic and minimally invasive surgery. The robotic surgical platform was introduced at the American University of Beirut Medical Center (AUBMC) in June 2013. Thirty-four consecutive PN procedures have been performed with no exclusion. The aim of this study was to compare the outcomes of open partial nephrectomy (OPN) 
Table 1. Demographic Data of Patients Undergoing Nephrectomy at the American University of Beirut Medical Center Between July 2013 and 2015

\begin{tabular}{llll} 
Variable & OPN & RAPN & P-value \\
\hline Tumor size, cm & $4.5 \pm 2.7$ & $3.6 \pm 1.7$ & 0.25 \\
RENAL score & $7 \pm 2$ & $5 \pm 1$ & $<0.05$ \\
Operative time, min & $178 \pm 52$ & $296 \pm 86$ & $<0.05$ \\
Ischemia time, min & Cold ischemia: $24 \pm 2$ & Warm ischemia: $17.5 \pm 1$ & Need for blood transfusions: 0 \\
Intraoperative blood loss, $\mathrm{mL}$ & $225 \pm 132$ & $243 \pm 192$ & 0.01 \\
Length of hospital stay, days & $6 \pm 1.6$ & $4 \pm 0.9$ & $<0.05$ \\
\hline change in GFR & $-9 \%$ & $-7 \%$ & \\
\hline
\end{tabular}

to RAPN during the initial introduction of robotic surgery at our institution.

\section{Materials and Methods}

After aquiring the institutional review board approval at the AUBMC, a retrospective chart review of all PNs, 19 OPNs and 15 RAPNs, was performed since the inception of the robotic program until July 2015. Preoperative patient demographics including patient age, sex, and tumor size were not controlled for among the two groups. Perioperative renal functional and patient-related outcomes (operative time, tumor complexity, RENAL score, intraoperative blood loss, ischemia time, length of hospital stay, estimated change in glomerular filtration rate (eGFR), and the need for blood transfusions) were charted, analyzed, and compared using Chi-square tests on SPSS, where a P-value $<0.05$ was considered significant.

\section{Results}

Thirty-four consecutive PNs were performed in two years: 19 OPN and 15 RAPN. Results are portrayed in Table 1.

\section{Tumor size and complexity}

The RENAL nephrometry score was used to assess complexity of the renal masses. A significantly higher score was obtained for masses assigned to the OPN group ( \pm 2 vs. $5 \pm 1, \mathrm{P}<$ $0.05)$. This however was not concordant with the size of the tumors as it was not significantly different among both groups $(4.5 \pm 2.7 \mathrm{~cm}$ in $\mathrm{OPN}$ vs. $3.6 \pm 1.7 \mathrm{~cm}$ in $\mathrm{RAPN}, \mathrm{P}=0.25)$.

\section{Intraoperative blood loss, need for blood transfusions, and eGFR}

Estimated blood loss was comparable among both approaches $(225 \pm 132 \mathrm{~mL}$ in OPN vs. $243 \pm 192 \mathrm{~mL}$ in RAPN, $\mathrm{P}<0.05)$ and there was no need for blood transfusions in either group. Likewise, the percent eGFR between preoperative and postoperative values was minimal and comparable among both groups $(\mathrm{OPN}=-9 \%$ vs. $\mathrm{RAPN}=-7 \%)$.

\section{Crude ischemia time}

All OPN cases were performed with arterial clamping and cooling using ice slush, unlike the RAPN cases which were performed with renal arterial clamping without cooling. The mean cold ischemia time was $24 \pm 2$ min, while the mean warm ischemia time was approximately $17.5 \pm 1 \mathrm{~min}$, and $10 / 15$ cases were performed in under $20 \mathrm{~min}$ of ischemia.

\section{Operative time and length of hospital stay}

The mean operative time was significantly shorter for OPN compared to RAPN (178 $\pm 52 \mathrm{~min}$ vs. $296 \pm 86 \mathrm{~min}$, respectively, $\mathrm{P}<0.05$ ), knowing that the time spent to dock the robot was subtracted when calculating operative time for the RAPN group. The length of hospital stay was longer for OPN vs. RAPN ( $6 \pm 1.6$ days vs. $4 \pm 0.9$ days, $\mathrm{P}<0.01)$ with no attributable postoperative complication to either procedure.

\section{Margin status and rate of conversion to radical nephrec- tomy}

None of the cases required conversion to radical nephrectomy. Moreover, the final pathological outcomes revealed one focally positive margin in the OPN group only.

\section{Discussion}

PN has been established as the gold standard for the surgical treatment of small renal masses as it preserves long-term renal function, and yields comparable oncologic control when compared to radical extirpative surgery $[1,2]$. The first landmark study on PN published by Novick and Licht in 1993, solidified OPN as a viable treatment modality $[4,5]$ for renal masses. However, with the advent of minimally invasive surgery, laparoscopic-assisted PN and RAPN have proven to be advantageous as they are associated with shorter length of hospital stay, diminished blood loss, and faster recovery with 
resumption of normal daily function $[2,6,7]$.

Since its introduction to our institution, RAPN has been increasingly applied for small amenable renal masses. The latter were stratified using the RENAL nephrometry score (RS) into low (4 - 6), moderate (7 - 9), and highly complex masses $(10-12)$. Our data show that $87 \%$ of all masses treated with RAPN (13/15) were of a low RS, while 58\% (11/19) of OPNs had a moderate/high RS. This may be due to the preference of robotic urologic surgeons to include non-complex masses in their initial series, thereby avoiding any mishaps during that learning period. One must acknowledge this complexity bias and its consequences on the final outcomes.

In a retrospective review comparing 81 RAPNs to 134 OPNs, Viterbo et al showed that the mean operative time for RAPN (205.9 $\pm 52.5 \mathrm{~min}, \mathrm{P}<0.01)$ is greater than that for OPN [8] (189.5 $\pm 52, \mathrm{P}<0.01)$; however, with increasing surgeon experience, more challenging renal tumors (e.g. central site or collecting system involvement) are currently robotically resected and with decreasing operative times [9]. At the AU$\mathrm{BMC}$, the operative time for RAPN is significantly longer than that of OPN $(296 \pm 86$ vs. $178 \pm 52 \mathrm{~min}, \mathrm{P}<0.005)$ which is most probably attributable to the surgeon's learning curve.

Intraoperative blood loss and complication rate were comparable among both procedures; the mean blood loss (EBL) was $235 \mathrm{cc}$ and there were no recorded complications among any of the 34 cases. In a multicenter retrospective study of 198 OPNs vs. 105 RAPNs, Minervini et al found a markedly lower EBL in RAPN $(125 \pm 128$ vs. $230 \pm 208, \mathrm{P}=0.004)$ and a lower overall rate of complication [10] $(8.6 \%$ vs. $24 \%, \mathrm{P}=$ $0.009)$ when compared to OPN. Such numbers were also portrayed by Lucas et al, who showed that EBL (100 mL vs. 250 $\mathrm{mL}, \mathrm{P}<0.001)$ and complication rates were lower for RAPN [11]. Our differing results are mostly attributable to the small sample size. With increasing surgeon experience and larger volumes, we may be able to decrease intraoperative blood loss and maintain low complication rates.

Multiple studies portray the advantages of RAPN particularly during the learning curve. Regarding periopearative complication rates, Ficarra et al reviewed 886 RAPNs in five different US-based centers, with a low mean intraoperative complication rate [12] of $2.5 \%$, comparable to outcomes at our institution. Other larger European retrospective reviews of more than 1,800 patients reveal a higher risk of complications from RAPN compared to our data; however, when compared to open surgery, RAPN remains advantageous with regard to its risk of perioperative complications (18\% vs. $28.6 \%, \mathrm{P}<$ $0.001)$ [13].

Another encouraging finding in favor of RAPN is the shorter length of hospitalization. Our data are in line with those of larger centers, in that the length of stay is significantly shorter in RAPN ( $4 \pm 0.9$ days vs. $6 \pm 1.6$ days, $\mathrm{P}=0.01)$. This may be attributed to the minimally invasive nature of the procedure, decreased postoperative pain, a faster convalescence and return to normal daily activities.

Several authors have written about the learning curve of RAPN. In a study of 150 RAPNs, Allaf et al showed that 25 consecutive cases are required to achieve an adequate transition from laparoscopic PN to RAPN [14]. However, one must note that, different surgical outcomes require a specific number of practiced cases to achieve, and hence a differing learning curve.

Mottrie et al have shown that a median operative time $<$ 100 min can be achieved after 20 consecutive cases, while a warm ischemia time $<20$ min needs roughly 30 cases to be achieved [15]. This comes to show that the learning curve per se is not an absolute value. It is calculated relative to the variable at stake. This has also been studied extensively by Haseebuddin et al, who evaluated the learning curver after 38 consecutive RAPNs and concluded that the learning curve for RAPN is short for surgeons already experienced with LPN [16]; however, as previously mentioned, when defined by the overall operative time, the learning curve for RAPN is shorter (16 cases), then when defined by ischemic time ( 26 cases). The robotic program at AUBMC has witnessed a slow start, with a larger number of cases ( $>30$ cases since July 2013) required to cut down the operative time. This may be attributed to the expertise of the surgeon, and the fact that the excess care that is taken to avoid complications (complication rate $0 / 15$ ) is time consuming.

\section{Conclusion}

RAPN seems to be a promising approach for the treatment of kidney tumors with the advantages of decreased crude ischemia time and a shorter hospital stay, with comparable intraoperative blood loss and risk of GFR reduction. Tumor characteristics were not equivalent, with higher RENAL scores noted in patients allocated to OPN vs. RAPN, thus limiting a fair comparison of outcomes. However, the data confirm that with proper selection of patients for RAPN, outcomes were equivalent to OPN and were not jeopardized during the robotic learning curve. Larger prospective studies are needed to validate our results.

\section{Conflicts of Interest}

The authors of this manuscript report no direct or indirect biomedical, financial, or other potential conflicts of interest.

\section{References}

1. Fergany AF, Hafez KS, Novick AC. Long-term results of nephron sparing surgery for localized renal cell carcinoma: 10-year followup. J Urol. 2000;163(2):442-445.

2. Miller DC, Schonlau M, Litwin MS, Lai J, Saigal CS. Renal and cardiovascular morbidity after partial or radical nephrectomy. Cancer. 2008;112(3):511-520.

3. Rogers CG, Singh A, Blatt AM, Linehan WM, Pinto PA. Robotic partial nephrectomy for complex renal tumors: surgical technique. Eur Urol. 2008;53(3):514-521.

4. Licht MR, Novick AC, Goormastic M. Nephron sparing surgery in incidental versus suspected renal cell carcinoma. J Urol. 1994;152(1):39-42.

5. Butler BP, Novick AC, Miller DP, Campbell SA, Licht MR. Management of small unilateral renal cell carcino- 
mas: radical versus nephron-sparing surgery. Urology. 1995;45(1):34-40; discussion 40-31.

6. Wu Z, Li M, Qu L, Ye H, Liu B, Yang Q, Sheng J, et al. A propensity-score matched comparison of perioperative and early renal functional outcomes of robotic versus open partial nephrectomy. PLoS One. 2014;9(4):e94195.

7. Wu Z, Li M, Liu B, Cai C, Ye H, Lv C, Yang Q, et al. Robotic versus open partial nephrectomy: a systematic review and meta-analysis. PLoS One. 2014;9(4):e94878.

8. Simhan J, Smaldone MC, Tsai KJ, Li T, Reyes JM, Canter D, Kutikov A, et al. Perioperative outcomes of robotic and open partial nephrectomy for moderately and highly complex renal lesions. J Urol. 2012;187(6):2000-2004.

9. Benway BM, Bhayani SB, Rogers CG, Dulabon LM, Patel MN, Lipkin M, Wang AJ, et al. Robot assisted partial nephrectomy versus laparoscopic partial nephrectomy for renal tumors: a multi-institutional analysis of perioperative outcomes. J Urol. 2009;182(3):866-872.

10. Minervini A, Vittori G, Antonelli A, Celia A, Crivellaro S, Dente D, Di Santo V, et al. Open versus robotic-assisted partial nephrectomy: a multicenter comparison study of perioperative results and complications. World J Urol. 2014;32(1):287-293.

11. Lucas SM, Mellon MJ, Erntsberger L, Sundaram CP. A comparison of robotic, laparoscopic and open partial nephrectomy. JSLS. 2012;16(4):581-587.

12. Ficarra V, Rossanese M, Gnech M, Novara G, Mottrie A. Outcomes and limitations of laparoscopic and robotic partial nephrectomy. Curr Opin Urol. 2014;24(5):441447.

13. Peyronnet B, Seisen T, Oger E, Vaessen C, Grassano Y, Benoit T, Carrouget J, et al. Comparison of 1800 Robotic and Open Partial Nephrectomies for Renal Tumors. Ann Surg Oncol. 2016;23(13):4277-4283.

14. Pierorazio PM, Patel HD, Feng T, Yohannan J, Hyams ES, Allaf ME. Robotic-assisted versus traditional laparoscopic partial nephrectomy: comparison of outcomes and evaluation of learning curve. Urology. 2011;78(4):813819.

15. Mottrie A, De Naeyer G, Schatteman P, Carpentier P, Sangalli M, Ficarra V. Impact of the learning curve on perioperative outcomes in patients who underwent robotic partial nephrectomy for parenchymal renal tumours. Eur Urol. 2010;58(1):127-132.

16. Haseebuddin M, Benway BM, Cabello JM, Bhayani SB. Robot-assisted partial nephrectomy: evaluation of learning curve for an experienced renal surgeon. J Endourol. 2010;24(1):57-61. 